\title{
Interventional oncology: state of the art
}

\author{
Gianpaolo Cornalba $\cdot$ Fabio Melchiorre
}

Received: 9 June 2014 / Accepted: 11 June 2014/Published online: 8 July 2014

(C) Italian Society of Medical Radiology 2014

Cancer is a major health problem in the Western world. Currently, approximately one in three people in the Western world will develop cancer in their lifetime and one in four will die of the disease. Over the years, much has been done on prevention and cure of this disease by surgery, chemotherapy and radiotherapy [1].

The landscape of medicine is constantly changing, and for the past 40 years, interventional radiologists have been responsible for much of the medical innovation and development of the minimally invasive procedures that are commonplace today. As innovative image-guided procedures have been developed, the interventional radiologist has become an integral member of a multidisciplinary approach to treating cancer. Radiologists can now be involved in all facets of care, including diagnosis, treatment, and imaging follow-up. Thus, interventional radiology (IR) can be inserted as the fourth pillar in tumour therapy [1].

Appropriate treatment of malignancy is dependent on a timely definitive diagnosis and on accurate staging of disease. While non-invasive imaging techniques have improved assessment and staging for cancer, histologic confirmation remains the gold standard for definitive diagnosis of many tumours. Biopsies to establish histological diagnosis are increasingly performed using minimally invasive techniques by interventional radiologists. These minimally invasive techniques are applicable to a wide range of biopsy sites and, in most organ systems, have

G. Cornalba $(\bowtie) \cdot$ F. Melchiorre

Università degli Studi di Milano, AO- San Paolo Milano, Milan, Italy

e-mail: gianpaolo.cornalba@unimi.it

F. Melchiorre

e-mail: fabio.melchiorre@ao-sanpaolo.it been demonstrated to be highly accurate with a low complication rate [2].

Minimally invasive image-guided cancer treatments as an adjunct or alternative to surgery are increasingly being used in the management of malignancy include transarterial embolisation, ablative techniques and gene therapy.

Transarterial embolisation can be used alone as the primary modality of treatment, where interruption of the afferent blood supply to the tumour induces hypoxia and inhibits tumour growth, or in conjunction with ablative treatments or conventional surgery. Transarterial chemoembolisation (TACE) is a modification of the above technique as is radioembolisation, a novel form of liverdirected brachytherapy [2].

Local tumour ablation is an alternative method of achieving tumour control in those patients with early-stage malignant disease, particularly in the liver, who are not candidates for resection. IR-mediated tumour ablation induces tumour necrosis by the application of energy and modalities employed include radiofrequency (RF), laser, microwave, ultrasound, electroporation and cryotherapy.

Last but not least, gene therapy has been made possible due to advances in molecular oncology and tumour immunology. In an IR technique similar to that used in chemoembolisation, genetic agents may be administered directly into the tumour mass by selective arterial injection, after which the vessel is embolised thus limiting adverse effects and prolonging agent dwell time which is believed to improve genetic transfer rate. Although there are few studies limited to small patient cohorts, this techniques is a promising one [3].

As one can observe IR has improved majorly in recent years and can be inserted as the fourth pillar in cancer therapy alongside surgery, chemotherapy and radiotherapy. In my opinion, the essence of the treatment modality of 
interventional oncology is technical skill, although it may also sometimes depend upon various kinds of devices and drugs. An expert interventional radiologist must also be prepared and informed on tumour pathology, other cancer treatments and patient management. Therefore, training the next generation of interventional radiologists is mandatory for the future of this branch.

Conflict of interest The authors declare no conflict of interest.

\section{References}

1. Kwee TC, Takahara T et al (2010) Cancer imaging: novel concepts in clinical magnetic resonance imaging. J Intern Med 268(2): $120-132$

2. Kevin AS, Hyun SK (2011) Interventional radiology and imageguided medicine: interventional oncology. Sem Oncol 38(1): $151-162$

3. Abi-Jaoudeh N, Duffy AG et al (2013) Personalized oncology in interventional radiology. J Vasc Interv Radiol 24(8):1083-1092 